\title{
Implementation of Smart Restaurant with e-menu Card
}

\author{
Mayur D. Jakhete \\ Asst. Prof; Electronics Dept; \\ G.H.R.I.E.M, Jalgaon \\ India
}

\author{
Piyush C. Mankar \\ M.E. student; Electronics Dept; \\ G.H.R.I.E.M, Jalgaon \\ India
}

\begin{abstract}
Today's era is said to be the world of technology. So many efforts have been taken by restaurants owners also to adopt information and communication technologies such as PDA, wireless LAN, costly multi-touch screens etc. to enhance dining experience. This paper highlights some of the limitations of the conventional paper based and PDA-based food ordering system and proposed the low cost touch screen based Restaurant Management System using an android Smartphone or tablet as a solution. The system consists of a Smartphone/tablet at the customer table contains the android application with all the menu details. The customer tablet, kitchen display connects directly with each other through WiFi. Orders made by the customers will be instantly reach the kitchen module. This wireless application is user-friendly, improves efficiency and accuracy for restaurants by saving time, reduces human errors and provides customer feedback. This system successfully overcomes the drawbacks in earlier automated food ordering systems and is less expensive as it requires a one-time investment for gadgets.
\end{abstract}

\section{Keywords}

Smartphone; automated; Wi-Fi; E-menu; android application; intelligent; ordering

\section{INTRODUCTION}

The advancement of Information and Communication Technology has led to an increasing number of industries to use electronic media and corresponding application for information exchange. In the restaurant sector, Modern wireless device such as Personal Digital Assistant (PDA) has been adopted into restaurant system to replace the conventional way of taking orders using pen and paper. However, the PDA-based food ordering system has known limitations such as the requirement of training of attendants, the need of having attendants to operate, the inefficiency during peak hours and small screen size and the Multitouchable restaurant Management System has limitations such as touch screens used are of mostly capacitive type or resistive type which are costly. Hence by introducing an application loaded in an android Smartphone or tablet containing the menu details these limitations can be overcome.

Android is a software stack for mobile devices that includes an operating system, middleware and key applications. Android boasts a healthy array of connectivity options, including Wi-Fi, Bluetooth, and wireless data over a cellular connection (for example, GPRS, EDGE (Enhanced Data rates for GSM Evolution), and 3G). Android provides access to a wide range of useful libraries and tools that can be used to build rich applications. In addition, Android includes a full set of tools that have been built from the ground up alongside the platform providing developers with high productivity and deep insight into their applications.

The project mainly aims in designing completely automated menu in restaurants with the help of Android mobile phone using Wi-Fi module and a LCD to provide a user-friendly environment. There is no need of a person to take the order from the table. The menu will be displayed automatically on the customer mobile application using wireless Wi-Fi connectivity and we can directly order the menu with the help of press on the menu.

\section{LITERATURE REVIEW}

The journey for getting up to the peak of joy and facilities that we are presently experiencing started with initial footstep of a wireless technology. The introduction of basic proposed systems and consequent developments are been mentioned here

Khairunnisa K. proposed the application of wireless food ordering system. This work presented in-depth on the technical operation of PDA[1] based Wireless Ordering System (WOS) including systems architecture, function, limitations and recommendations.

N. M. Z. Hashim presented an approach to develop a system by introducing the integration of Bluetooth technology[2] as the communication medium and Peripheral Interface Controller (PIC) as the hardware which implemented faster ordering system.

S. R. Patil, Snehal Salunkhe highlighted the limitations of the existing technologies and proposed the advanced system, which focuses on low cost touch-screen[3] development to enhance the dining experience.

K. A. Wadile developed a control system for autonomous mobile robots[4] used in Hotel management. Mobile robot having minimal centralized control was developed. The work focused on the development of two basic motion control algorithms, namely a GOTO algorithm and a FOLLOW algorithm, for use in a master-slave system. These robot motion control algorithms would have wide applicability in hotel operations.

Ashwini Bankar, Mamta Mahajan later on took one step ahead. They further exended the service with Paypal[5]. Technologies were used innovatively in a modern restaurant such as multi-touch LCD with Arduino mega, RF module, database \& line following Robot to enhance quality of services and to enrich customers' dining experience. 
Sushmita Sarkar presented the integration of touch technology in restaurants using android. This system was a basic dynamic database utility system which fetches all information from a centralized database[6]. The tablet at the customer table contains the android application with all the restaurant and menu details.

Suradej Sarmaputra furthermore put the enchanting concept of NFC i.e. Near field Communication for restaurant field[7]. The solution "Food Pre-Order System using NFC Based Smartphone" introduced an alternative method for the guests to be able to create the order before they approach the restaurant. Using NFC based Smartphone; the pre-ordered items can be captured by touching the phone at the NFC attached menu poster placed in eye-catching crowded area. When the guests approach the restaurant, the saved order can be confirmed by touching the Smartphone at the Order Dispatching Station located in the service area inside the restaurant.

Kiran Kumar reddy, B.Naresh employed combination of Bluetooth technology along with android phone[8]. An android application was designed containing food item details in restaurant. The input device was Smartphone or tablet and output section was PC. Cloud-based server for storing the database was used which made it inexpensive \& secure.

Asan, N. Badariah introduced Smart Ordering System which was also a fast way to order food at a restaurant. The system used a small keypad to place orders and the order made by inserting the code on the keypad menu. These codes came along with the menu. The signal would be delivered to the order by the Zigbee technology, and it would automatically be displayed on the screen in the kitchen.[9].

M. Firdouse Ali Khan and V. Swapna later on added facility like speech commands and wireless technology like Zigbee for data transmission from input section i.e. customer to kitchen section[10]. The system would automatically complete data receiving, storage, display, and analysis. It was provided with many advantages as great flexibility, portability

\section{PROPOSED WORK}

We propose this integration of touch technology in restaurants based on android technology. It is a wireless food ordering system using android devices. Android devices, in the past few years, have reached the pinnacle of popularity and have revolutionized the use of mobile technology in the automation of routine task in wireless environment.

\subsection{System block diagram}

The system block diagram of Touch and Order in restaurants is shown in figure. The android application on tablets at the tables. The tablets will be provided to customers, at their tables, allowing them to directly view the menu card and order immediately from their respective tablets. The tablets are the property of the establishment and are kept at each table.

This system, however, is confined within the restaurant vicinity only.

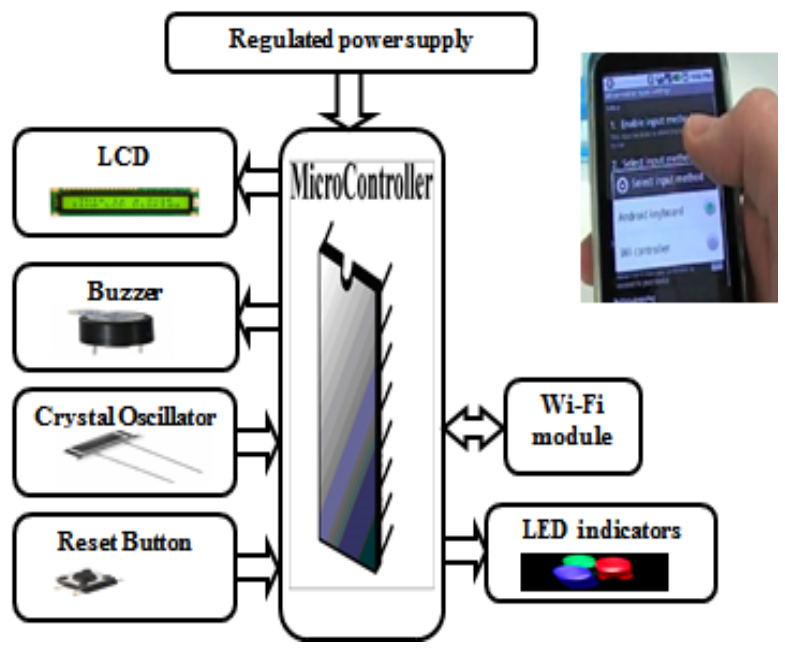

Fig 1. System block diagram

\subsection{System Overview}

The microcontroller used in this project is ARM LPC2148. It is a 64 pin Microcontroller which comes under ARM 7 version of ARM controllers. This is intended for high end applications involving complex computations. It follows the enhanced RISC architecture. It has high performance and very low power consumption.

Reset is used for putting the microcontroller into a 'known' condition. That practically means that microcontroller can behave rather inaccurately under certain undesirable conditions. In order to continue its proper functioning it has to be reset.

The LPC 2148 board readily offers crystal oscillator with $60 \mathrm{Mhz}$ frequency which leads to excellent system performance. The LEDs are used for indication purpose. WiFi module shows connectivity with Smartphone through small LED on it

Wi-Fi is a wireless technology that uses radio frequency to transmit data through the air. Wi-Fi has initial speeds of $1 \mathrm{mbps}$ to $2 \mathrm{mbps}$. Wi-Fi transmits data in the frequency band of $2.4 \mathrm{GHz}$. It implements the concept of frequency division multiplexing technology. Range of Wi-Fi technology is $40-300$ feet.

A liquid crystal display (LCD) is a thin, flat electronic visual display that uses the light modulating properties of liquid crystals. Liquid crystal display is very important device in embedded system. It offers high flexibility to user as he can display the required data on it.

Here we have used it for displaying the order at kitchen section.

A buzzer or beeper is an audio signaling device, which may be mechanical, electromechanical, or electronic. It will sound for indicating that a new order is been received at kitchen section.

Fig. shown below is the actual circuit diagram for our system: 


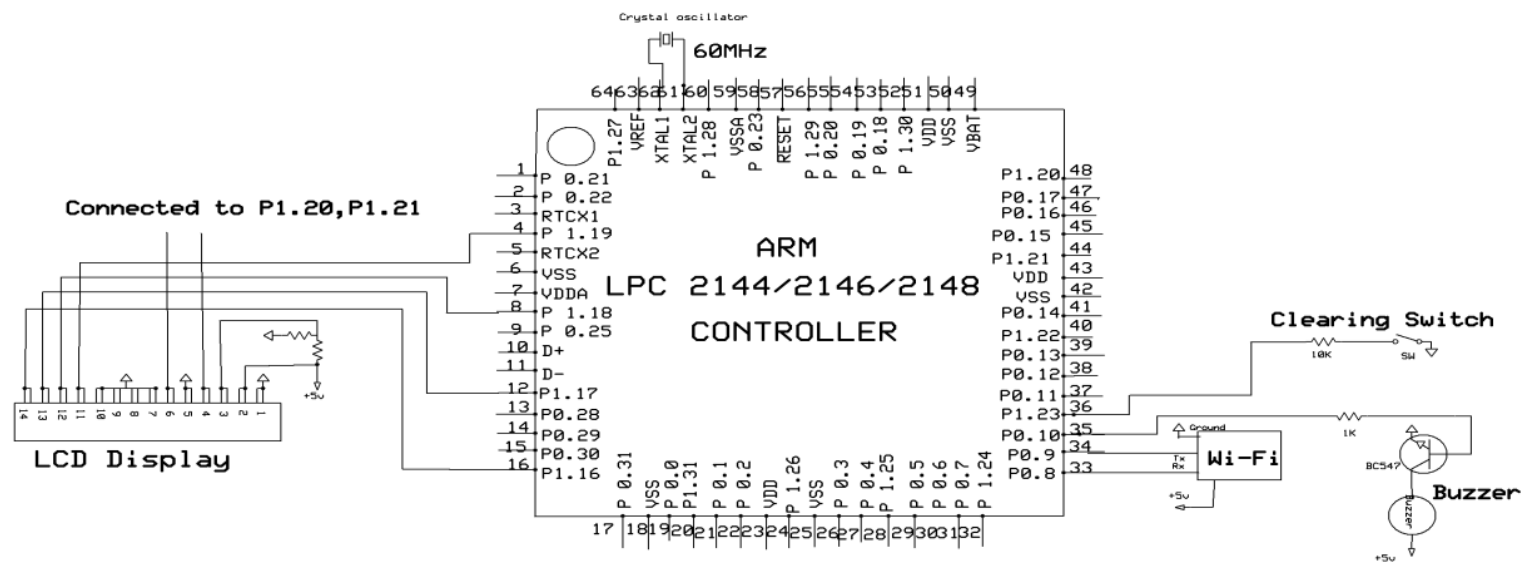

Fig 2. Circuit diagram for system

\subsection{Methodology}

Each table in the restaurant will be accompanied with an android tablet or a Smartphone. The device will be loaded with an android supporting application containing food menu details at that restaurant.

All the above mentioned digital components such as Smartphone/tablet (containing menu app), Microcontroller, an another android phone (we used it for enabling hot-spot to tether the wireless network) and Wi-Fi module are connected wirelessly to create a Wi-Fi network.

Some steps are mentioned here to understand the working of the system easily:

- Enable the Wi-Fi on Smartphone/tablet on which you wish visualize the E- menu card.

- Turn on the Portable hotspot on another android phone to tether wireless network.

- Now switch on the power supply. We will find an initial indication of buzzer and LED. (The blinking small LED on Wi-Fi module will stop blinking immediately after detecting the Wi-Fi on Smartphone/tablet.)

- Now we can observe the connected devices (Wi-Fi module and Smartphone containing app) on the phone on which hotspot is enabled.)

- As we tap to open the application that we have designed for menu details, we will be asked to enter the IP address and port number of system.

- After entering the valid credentials we will be presented with the screen in that Smartphone/tablet containing beautifully colored images of the menus in the restaurant.

- Person may tap on the image that he/she want to order. After clicking on, command will go to microcontroller through Wi-Fi.

- The microcontroller is dumped with an embedded C coding containing all the details regarding menu.

- Eventually it will command to the LCD to display the corresponding order on it at the kitchen section along with buzzer indication.
Flowchart for understanding the system operation.

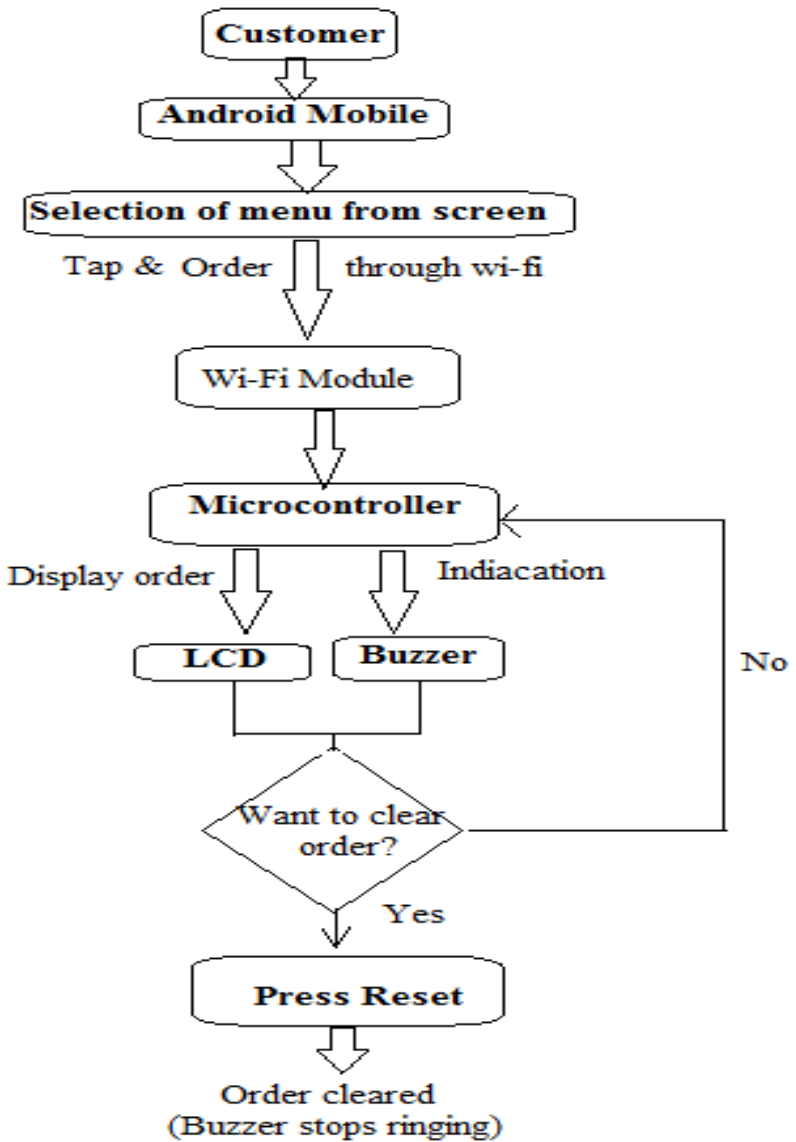

Fig 3. System Operation

\subsection{Technologies}

This project is implemented using following software's:

\subsubsection{Express $P C B-$}

Express PCB is a software tool to design PCBs specifically for manufacture by the company Express PCB (no other PCB maker accepts Express PCB files). It is very easy to use 


\subsubsection{Keil $\mu$ Vision4 software -}

The $\mu$ Vision IDE from Keil combines project management, make facilities, source code editing, program debugging, and complete simulation in one powerful environment. The $\mu$ Vision development platform is easy-to-use and helping you quickly create embedded programs that work. The $\mu$ Vision editor and debugger are integrated in a single application that provides a seamless embedded project development environment.

\subsection{Assumptions}

The success of the proposed system is based on the assumptions that:

- End users should be able to use tablets.

- Network connection will always remain stable.

- Tablets should work fault free.

\subsection{Advantages}

- Low power consumption.

- No need of a person to take order from the table.

- Long life.

- Highly sensitive

- Easy to install because of wireless interface.

- Usage of Android touch screen smart phone in performing the task.

- Wi-Fi wireless transmission

- Useful for even illiterates and dumb people.

- Can be used with any language.

- $\quad$ Easy to install because of wireless interface.

- Fast response.

- Efficient and low cost design.

- Low power consumption.

\subsection{Limitations:}

- Status and feedback of order is not obtained.

- $\quad$ Limited distance (Generally confined to a hall).

- System may not work properly if tablet/Smartphone suffer a defect

- Also may become a drawback if end users are not able to use the tablet/Smartphone devices.

\section{TEST RESULTS}

Following are the screenshots of actual results of our system.

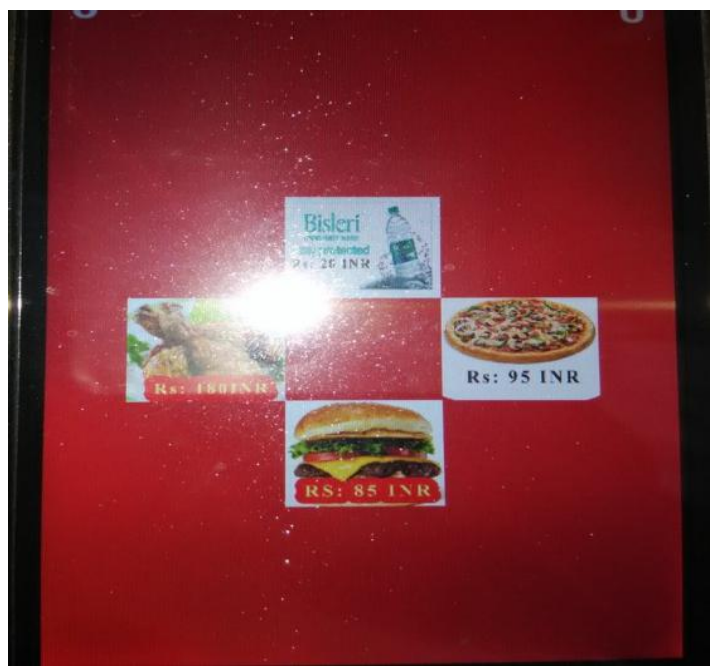

Fig 4. Menu options at customer mobile.

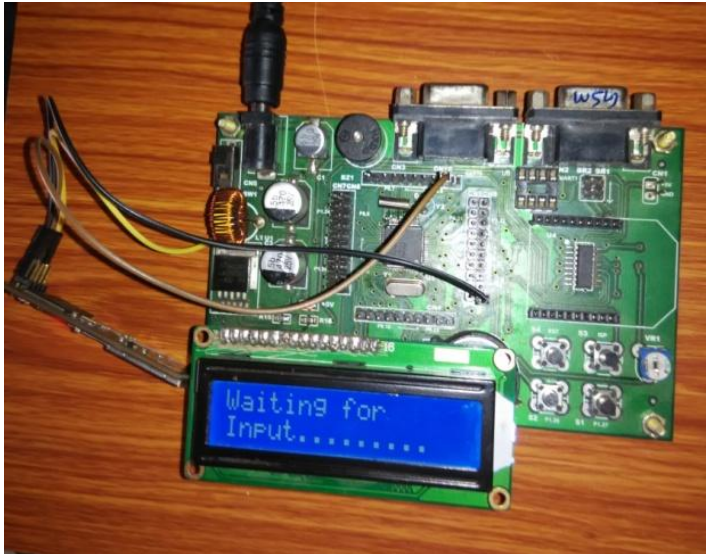

Fig 5. Status when no input (order) is given

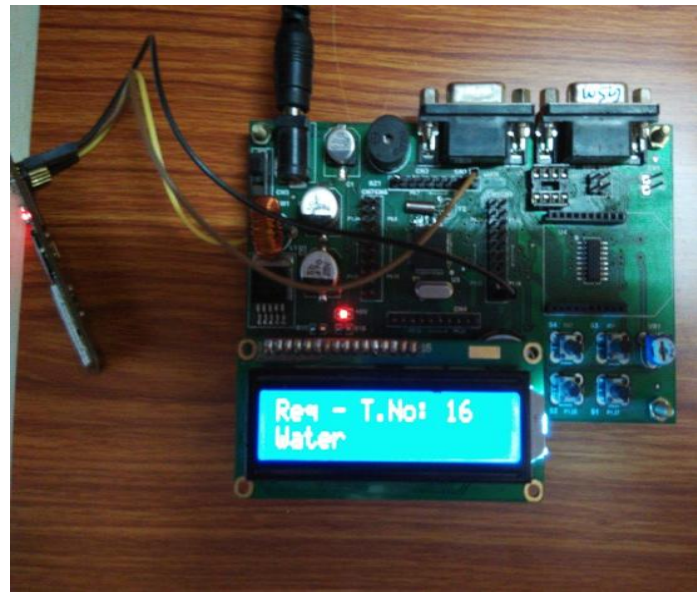

Fig 6. Order received for Water

\section{CONCLUSION \& FUTURE SCOPE}

Integrating features of all the hardware components used have been developed in it. Presence of every module has been reasoned out and placed carefully, thus contributing to the best working of the unit. Secondly, using highly advanced IC's with the help of growing technology, the project has been successfully implemented. Smart restaurant is developed in order to provide easy interaction between customers through wireless technology. Thus the project has been successfully designed and tested.

Similarly, the system can also be implemented with Graphical LCD for displaying the menu as we have used android phone. However the system becomes more bulky and delicate to handle because each table is going to consist of such module for ordering.

\section{REFERENCES}

[1] Khairunnisa K." The Application of Wireless Food Ordering System" in MASAUM Journal of Computing, Volume 1 Issue 2, September 2009

[2] N. M. Z. Hashim "Smart Ordering System via Bluetooth" in International Journal of Computer Trends and Technology (IJCTT) - volume 4 Issue 7-Month 2013

[3] S. R. Patil "e-converse An affordable touch screen solution to intrigue Dining experience" 
[4] K. A. Wadile "E- restaurant management system using robot" in international journal of informative \& futuristic research. 2015

[5] Ashwini Bankar "Review paper on - Design of Intelligent Restaurant with a Touch Screen Based Menu

[6] Sushmita Sarkar "Integration of Touch Technology in Restaurants using Android" in International Journal of Computer Science and Mobile Computing 2014

[7] Suradej Sarmaputra," The Food Pre-Order System for Restaurant using NFC Based Smartphone" Technopreneurship and Innovation Management Program Chulalongkorn University..
[8] Kiran Kumar reddy, B.Naresh"Intelligent E-Restaurant using android OS" international journal of scientific engineering and technology 2014

[9] Asan, N. Badariah" Zigbee-Based Smart Ordering System (S.O.S)" International Journal of Computer Trends and Technology (IJCTT) - volume 11 number 5 - May 2014

[10] M. Firdouse Ali Khan and V. Swapna" Design And Implementation Of Ordering System For Restaurants" in International Journal of Engineering Research \& Technology (IJERT) in 2012 